Box Dimension and Cyclicity of Canard Cycles

Peer-reviewed author version

HUZAK, Renato (2018) Box Dimension and Cyclicity of Canard Cycles. In:

Qualitative Theory of Dynamical Systems, 17 (2), p. 475-493.

DOI: $10.1007 / \mathrm{s} 12346-017-0248-\mathrm{x}$

Handle: http://hdl.handle.net/1942/24036 


\title{
Box dimension and cyclicity of canard cycles
}

\author{
Renato Huzak \\ Hasselt University, Campus Diepenbeek, Agoralaan Gebouw D, \\ 3590 Diepenbeek, Belgium
}

\begin{abstract}
It is well known that the slow divergence integral is a useful tool for obtaining a bound on the cyclicity of canard cycles in planar slow-fast systems. In this paper a new approach is introduced to determine upper bounds on the number of relaxation oscillations Hausdorff-close to a balanced canard cycle in planar slow-fast systems, by computing the box dimension of one orbit of a discrete one-dimensional dynamical system (so-called slow relation function) assigned to the canard cycle.
\end{abstract}

\section{Introduction}

Studying smooth or analytic $\lambda$-families of planar vector fields $\{\dot{x}=f(x, y, \lambda), \dot{y}=$ $g(x, y, \lambda)\}, \lambda \in \mathbb{R}^{p}$, one often encounters so-called limit periodic sets, at level $\lambda_{0}$, that can generate limit cycles for $\lambda \neq \lambda_{0}$ and $\lambda \sim \lambda_{0}$. The maximum number of limit cycles Hausdorff close to a monodromic limit periodic set, i.e. a limit periodic set accumulated on one side by spiral trajectories (e.g. foci, limit cycles, homoclinic loops,...), can be found by studying fixed points of Poincaré maps $P_{\lambda}$ defined arround the monodromic limit periodic set. Following [EŽŽ07] and [ŽŽ08], fractal analysis of the trajectories that spiral around a focus or a limit cycle is a useful tool for finding out how many limit cycles can appear in bifurcations close to the focus or the limit cycle. More precisely, the density of an arbitrary but fixed orbit of the Poincare map $P_{\lambda_{0}}$ near the fixed point, corresponding to the focus or the limit cycle, tells us the maximum number of limit cycles, i.e. the cyclicity. The density is typically measured by estimating the length of $\delta$-neighborhood of the orbit as $\delta \rightarrow 0$, and comparing the length with $\delta, \sqrt[2]{\delta}, \sqrt[3]{\delta}, \ldots$ In this way one obtains the so-called box dimension of the orbit, and it lies between 0 and 1 . The bigger the box dimension of the orbit (i.e. the density of the orbit), the more limit cycles can be born for $\lambda \sim \lambda_{0}$. For more details see Section 2. In [MRŽ12], these results have been generalized to a broader class of monodromic limit periodic sets around which the Poincaré maps are non-differentiable (e.g. homoclinic loops, Hamiltonian 2-saddle cycles with constant hyperbolicity ratios, ...) by introducing the notion of the critical Minkowski order. Note that the notion of box dimension used in [EŽŽ07, ŽŽ08] is close to the notion of the Hausdorff dimension usually used for measuring the complexity of fractal sets in dynamics. Nevertheless, the orbits of the Poincaré map are countable sets accumulating at the fixed point. Their Hausdorff dimension is trivial due to its countable stability property. On the other hand, their 
box dimension is nontrivial and measures the local density of accumulation at the fixed point.

The purpose of this paper is to apply the box dimension approach to so-called slow-fast limit periodic sets, containing a curve of singularities, that appear in planar slow-fast systems. The Poincaré map $P_{\lambda_{0}}$ is not defined near such (nonmonodromic) slow-fast limit periodic sets and therefore we cannot estimate asymptotic behavior of the length of $\delta$-neighborhood of an orbit of $P_{\lambda_{0}}$ to obtain the cyclicity. As we will see in later sections, it is the slow relation function/slow divergence integral calculated along the curve of singularities that enables us to define a suitable discrete one-dimensional dynamical system that plays the role of the Poincaré map $P_{\lambda_{0}}$.

A smooth planar slow-fast system is locally given by

$$
\left\{\begin{array}{l}
\dot{x}=f(x, y, \epsilon, \mu) \\
\dot{y}=\epsilon g(x, y, \epsilon, \mu)
\end{array}\right.
$$

where $\epsilon \geq 0, \epsilon \sim 0$ is the singular perturbation parameter, $\mu \in \mathbb{R}^{p}$, for some $p \geq$ 1 , and $f$ and $g$ are smooth functions. In this paper "smooth" means $C^{\infty}$-smooth. When $\epsilon=0$, we call (1) the layer equation or the fast subsystem consisting of (fast) regular horizontal orbits, and a curve of singularities $\{f(x, y, 0, \mu)=0\}$, called the critical curve or the slow curve, for each $\mu$. See Fig. 1. We suppose that all singularities of the slow curve are normally hyperbolic (attracting or repelling), except finitely many of singularities, so called contact points, each having both eigenvalues equal to zero. The qualitative behavior of the system (1) near the slow curve (away from the contact points) is usually studied by using so called slow dynamics $y^{\prime}=g(x, y, 0, \mu)$ along the slow curve (we will give a precise definition of "slow dynamics" in later sections).

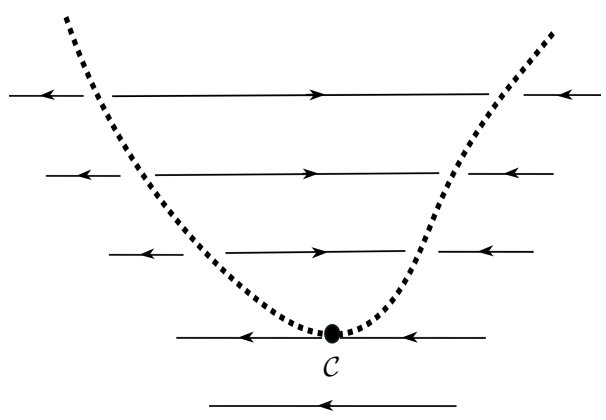

Figure 1: A possible layer equation of (1), with a contact point $\mathcal{C}$.

For $(\epsilon, \mu)=\left(0, \mu_{0}\right)$ we distinguish between two types of limit periodic sets that can give rise to a number of limit cycles in their Hausdorff neighborhood for $(\epsilon, \mu) \sim\left(0, \mu_{0}\right)$, slow-fast cycles and contact points. The slow-fast cycles consist of fast orbits of the layer equation and parts of the slow curve, and they can produce so called relaxation oscillations in perturbations. See e.g. [Ben81a, DR96, DR01, DR07, Dum11]. A slow-fast cycle is said to be canard if it contains both attracting and repelling parts of the slow curve. Otherwise, the slow-fast cycle is said to be common (see e.g. [DMDR11]). The contact points can be of very diverse nature: jump points (see e.g. [DR96, MKKR94]), slow-fast Hopf 
points (often called generic turning points) (see [DR96, KS01, DR09]), slowfast Bogdanov-Takens points (see [DMD11b]), slow-fast codimension 3 saddle or elliptic points (see [HDMD13, HDMD14, Huz16]), slow-fast codimension 4 saddle-node points (see [Huz17]), etc. Periodic orbits of (1), for $\epsilon>0$, Hausdorff close to jump points are not possible but a slow-fast cycle may contain jump points near which limit cycles, Hausdorff close to the slow-fast cycle, follow an attracting part of the slow curve and continue close to a fast orbit. Contact points are called turning points if they allow the passage from an attracting part of the slow curve to a repelling part of the slow curve, for $\epsilon>0$. The turning points can generate limit cycles in perturbations or can be contained in canard slow-fast cycles near which relaxation oscillations may appear (e.g. slow-fast Hopf points, slow-fast codimension 3 elliptic points, etc.).

In this paper, our main focus is on canard (slow-fast) cycles consisting of a fast orbit and the part of the slow curve between the $\alpha$-limit set and the $\omega$-limit set of the fast orbit (see Fig. 1). The part of the slow curve consists of one attracting part, one repelling part, and a single turning point. The most important tool for studying the cyclicity of such a canard cycle is the slow-divergence integral computed along the "slow part" of the canard cycle (see e.g. [DR96, DR01, DMD05, DMD08, DMD10, DMD11a]). Our goal is to introduce a new method for finding maximum number of relaxation oscillations Hausdorff close to so-called balanced canard cycles (roughly speaking, a canard cycle is balanced if the slow divergence integral calculated along the slow part of the canard cycle is zero) that consists in computing the box dimension of one orbit of the so-called slow relation function, defined close to the balanced canard cycle. See Section 3. To find (one orbit of) the slow relation function, we don't need to compute the slow divergence integral (see e.g. [Dum11] or Section 3). Thus, this method could be useful in applications when it is difficult to calculate the slow divergence integral.

For the sake of readability, in this paper we apply the fractal theory [EŽŽ07, ŽŽ08, MRŽ12] to a special smooth family of planar slow fast systems given in (3). Near the origin $(x, y)=(0,0)$, the system (3), with \pm in front of the term $x^{2 n-1}$, is a smooth normal form for equivalence for slow-fast systems which have a slow curve with a generic nilpotent contact point and a singularity of order $2 n-2$ in the slow dynamics located at the contact point (see e.g. [DMDR11]). (A nilpotent contact point is generic if there is a quadratic contact between the slow curve and the fast orbit, at the nilpotent contact point.) Since we will focus on canard cycles in (3), we suppose (3) is defined in a (large) closed disk with center $(0,0)$ in the $(x, y)$-plane. When we have the + sign in front of $x^{2 n-1}$ in (3), limit cycles near the canard cycles are not possible because the slow dynamics in that case does not allow the passage from the attracting slow curve to the repelling slow curve (see [DMD11a] or Section 3).

We point out that the "box dimension" method we present in this paper in the special framework of the slow-fast systems (3) can be used in a more general framework of [DR96, KS01, DMD05, DMD08, Dum11, DMD10, DMD11a] (for more details see Section 3).

When $n=1$, the system (3) has a slow-fast Hopf point at the origin. The number of limit cycles near the Hopf point or canard cycles depends on the higher order terms in $H(x, \mu)$. In a codimension 1 Hopf case, limit cycles near these two types of limit periodic sets have been studied in [DR96, KS01]. In 
higher codimensions, limit cycles near the Hopf point (resp. the canard cycles) have been studied in [DR09] (resp. in [DMD05], if the slow dynamics is regular, and in [DMD08], if the slow dynamics has isolated singularities (away from the slow-fast Hopf point)). As mentioned above, the slow divergence integral has been used to study the limit cycles near the canard cycles in the slow-fast Hopf case. When the slow dynamics in the slow-fast Hopf case is regular, we obtain a bound on the cyclicity of a balanced canard cycle in terms of the box dimension of a slow relation function (see Theorem 2 in Section 3.1). If the box dimension of the slow relation function is 0 (or equivalently, the slow relation function has a hyperbolic fixed point) or $\frac{1}{2}$ (in this case the slow relation function can undergo a saddle-node bifurcation of fixed points), we describe limit cycle bifurcations that occur (Hausdorff) close to the balanced canard cycle, with and without breaking parameter $b_{0}$ (see Theorems 3-4 in Section 3.1). In the proof of Theorems 3-4, we use Theorem 1 of Section 2 and the results obtained in [Dum11] for generic Hopf breaking mechanisms. Following [Dum11], the system (3), with $n=1$ and regular slow dynamics, is an example of the generic Hopf breaking mechanism. When the slow dynamics has singularities, we cannot define the slow relation function, and we obtain a bound on the cyclicity in terms of the "box dimension of the derivative of the slow divergence integral" (see Theorem 5 of Section 3.1)

A bound on the cyclicity of canard cycles in (3), with $n=2$ (resp. $n \geq 3$ ) is given in terms of the derivative of the slow divergence integral in [DMD10] (resp. [DMD11a]). For more details see Section 3.2. Like in Theorem 5, we express the bound on the cyclicity in terms of the "box dimension of the derivative of the slow divergence integral" (see Theorem 6).

In Section 2 we recall some parts of the box dimension approach [EŽŽo7, ŽŽ08, MRŽ12] which will be relevant for our paper. In Section 4 we prove Theorems 2-6.

\section{Box dimension of bounded sets}

In this section we recal the definition of the Minkowski content and the box dimension of a bounded set in $\mathbb{R}^{n}$ from e.g. [Mat95, Fed69, KP99, Fal90], and the result obtained in [EŽŽ07, MRŽ12], for $n=1$, that connects the multiplicity of an isolated fixed point of a smooth function, defined near the fixed point, with the box dimension of any orbit accumulating at the fixed point.

We take a bounded set $U \subset \mathbb{R}^{n}$ and denote by $U_{\delta}$ the $\delta$-neighborhood of $U$ : $U_{\delta}=\left\{x \in \mathbb{R}^{n} \mid d(x, U) \leq \delta\right\}$. Furthermore, we denote by $\left|U_{\delta}\right|$ the Lebesgue measure of $U_{\delta}$. The fractal properties of $U$ are closely related to the behavior of $\left|U_{\delta}\right|$ when $\delta \rightarrow 0$. The rate at which $\left|U_{\delta}\right|$ decreases, as $\delta \rightarrow 0$, reveals the density of accumulation of the set in the ambient space $\mathbb{R}^{n}$. It is measured by the box dimension (the upper box dimension is sometimes called the limit capacity) and the Minkowski content of $U$. First, we define the lower s-dimensional Minkowski content of $U$ (resp. the upper $s$-dimensional Minkowski content of $U$ ), $0 \leq s \leq n$, by

$$
\mathcal{M}_{*}^{s}(U)=\liminf _{\delta \rightarrow 0} \frac{\left|U_{\delta}\right|}{\delta^{n-s}}\left(\operatorname{resp} . \mathcal{M}^{* s}(U)=\limsup _{\delta \rightarrow 0} \frac{\left|U_{\delta}\right|}{\delta^{n-s}}\right) .
$$

Now, we can define the lower box dimension of $U$ (resp. the upper box dimension 
of $U)$ by

$\underline{\operatorname{dim}}_{B} U=\inf \left\{s \geq 0 \mid \mathcal{M}_{*}^{s}(U)=0\right\} \quad\left(\right.$ resp. $\left.\overline{\operatorname{dim}}_{B} U=\inf \left\{s \geq 0 \mid \mathcal{M}^{* s}(U)=0\right\}\right)$.

When $\operatorname{dim}_{B} U=\overline{\operatorname{dim}}_{B} U$, then we denote it by $\operatorname{dim}_{B} U$ and call it the box dimension of $U$. For properties of box dimension, see e.g. [Fal90].

Our ambient space has dimension one, i.e. $n=1$. Let $F$ be a smooth function on [0, $x_{*}\left[\right.$, for $x_{*}$ positive and small, $F(0)=0,0<F(x)<x$, for each $x \in] 0, x_{*}[$. Let

$$
G(x):=x-F(x) .
$$

Let $U=\mathcal{O}_{x_{0}}^{G}:=\left\{x_{n}=G^{n}\left(x_{0}\right) \mid n \in \mathbb{N}\right\}$, where $\left.x_{0} \in\right] 0, x_{*}[$, be the orbit of $x_{0}$ by $G$. Then $x_{n}$ tends monotonically to zero. It has been shown in [EŽŽ 07 ] that $\operatorname{dim}_{B} \mathcal{O}_{x_{0}}^{G}$ is independent of the initial point $x_{0}$, and one can define the box dimension of $G$ : $\operatorname{dim}_{B} G:=\operatorname{dim}_{B} \mathcal{O}_{x_{0}}^{G}$, for any $\left.x_{0} \in\right] 0, x_{*}[$.

We say that the multiplicity of the fixed point 0 of the smooth $G$ is equal to $m$, and write $m_{0}^{f i x}(G)=m$, if $x=0$ is a zero of multiplicity $m$ of $F$, i.e. $F(0)=\cdots=F^{(m-1)}(0)=0$ and $F^{(m)}(0) \neq 0$. We say that the multiplicity of the fixed point 0 of $G$ is $\infty$ if $F^{(n)}(0)=0$, for all $n \in \mathbb{N}$. If $F_{1}(x)$ and $F_{2}(x)$ are two positive functions defined for $x>0$ and $x \sim 0$, we write $F_{1}(x) \simeq F_{2}(x)$ as $x \rightarrow 0$ if $A F_{2}(x) \leq F_{1}(x) \leq B F_{2}(x)$, for two positive constants $A$ and $B$, and for any $x>0$ and $x \sim 0$.

The following theorem plays a crucial role in this paper.

Theorem 1 ([EŽŽ07, MRŽ12]). Let $F$ be a smooth function on $\left[0, x_{*}[\right.$, positive and nondecreasing on $] 0, x_{*}\left[\right.$ and $F(0)=0$. Put $U=\mathcal{O}_{x_{0}}^{G}$, with $G=i d-F$ and $x_{0} \in\left[0, x_{*}[\right.$.

If $1<m_{0}^{f i x}(G)<\infty$ (i.e. $G$ has a nonhyperbolic fixed point at 0 ), then

$$
\left|U_{\delta}\right| \simeq \delta^{\frac{1}{m_{0}^{f i x}(G)}} \text {, as } \delta \rightarrow 0 .
$$

If $m_{0}^{\text {fix }}(G)=1$ and $F(x)<x$ on $] 0, x_{*}[$ (i.e. $G$ has a hyperbolic fixed point at 0$)$, then

$\left|U_{\delta}\right| \simeq\left\{\begin{array}{ll}\delta(-\log \delta), & F^{\prime}(0)<1(\text { the "standard" hyperbolic case }), \\ \delta \log (-\log \delta), & F^{\prime}(0)=1(\text { the "degenerate" hyperbolic case }),\end{array}\right.$ as $\delta \rightarrow 0$.

For $1 \leq m_{0}^{f i x}(G)<\infty$, a bijective correspondence holds

$$
m_{0}^{f i x}(G)=\frac{1}{1-\operatorname{dim}_{B} G} .
$$

If $m_{0}^{\text {fix }}(G)=\infty$, then $\operatorname{dim}_{B} G=1$.

Proof. Theorem 1 has been proved in [EŽŽ07] (Theorems 1 and 6) or in [MRŽ12] (Theorem 1). In order to give a flavor of the methods used in [EŽŽ07, MRŽ12], we sketch the proof of Theorem 1 in two special cases: $1 . F(x)=x-x^{2}$ (the hyperbolic fixed point case), 2. $F(x)=x^{2}$ (the nonhyperbolic fixed point case).

In both cases, for every $\delta \sim 0$ and $\delta>0$, we decompose the $\delta$-neighborhood $U_{\delta}$ of $U=\mathcal{O}_{x_{0}}^{G}$ into two parts, the nucleus $N_{\delta}$ and the tail $T_{\delta}$ (see Fig. 2). This 
method of estimating the length of the $\delta$-neighborhood as $\delta \rightarrow 0$ by decomposing it into tail and nucleus is taken from [Tri95]. The tail $T_{\delta}$ is the union of $\delta$ neighborhoods of the points $x_{0}, x_{1}, \ldots, x_{n_{\delta}-1}$. The index $n_{\delta} \in \mathbb{N}$ is the smallest index such that the $\delta$-neighborhood of $x_{n_{\delta}}$ and the $\delta$-neighborhood of $x_{n_{\delta}+1}$ have non-empty intersection. The index $n_{\delta}$ is well-defined, and the $\delta$-neighborhood of $x_{n}$ and the $\delta$-neighborhood of $x_{n+1}$ have non-empty intersection for each $n \geq n_{\delta}$, because the sequence $\left(x_{n}-x_{n+1}\right)_{n \in \mathbb{N}}=\left(F\left(x_{n}\right)\right)_{n \in \mathbb{N}}$ tends monotonically to zero. Thus, we have $\left|U_{\delta}\right|=\left|T_{\delta}\right|+\left|N_{\delta}\right|,\left|T_{\delta}\right|=n_{\delta} 2 \delta \simeq n_{\delta} \delta$, as $\delta \rightarrow 0$, and $\left|N_{\delta}\right|=x_{n_{\delta}}+2 \delta$.

1. $F(x)=x-x^{2}$. Thus $G(x)=x^{2}, m_{0}^{f i x}(G)=1$ and $F^{\prime}(0)=1$. Moreover, we explicitely have $x_{n}=G\left(x_{n-1}\right)=x_{0}^{2^{n}}, n \geq 0$.

To estimate $n_{\delta}$ and $x_{n_{\delta}}$ as $\delta \rightarrow 0$, we use $2 \delta \simeq\left(x_{n_{\delta}}-x_{n_{\delta}+1}\right)=F\left(x_{n_{\delta}}\right)=$ $x_{n_{\delta}}-x_{n_{\delta}}^{2} \simeq x_{n_{\delta}}=x_{0}^{2^{n_{\delta}}}$, as $\delta \rightarrow 0$. This implies that $n_{\delta} \simeq \log (-\log \delta)$ and $x_{n_{\delta}} \simeq \delta$, as $\delta \rightarrow 0$. Thus, we obtain

$$
\left|T_{\delta}\right| \simeq \delta \log (-\log \delta),\left|N_{\delta}\right| \simeq \delta, \delta \rightarrow 0
$$

Now it can be easily seen that $\left|U_{\delta}\right| \simeq \delta \log (-\log \delta)$, as $\delta \rightarrow 0$, and $\operatorname{dim}_{B} G=0$. Note that the estimates above and the box dimension do not depend on the choice of the initial point $x_{0}$ of the orbit.

2. $F(x)=x^{2}$. Then $G(x)=x-x^{2}$ and $m_{0}^{f i x}(G)=2$. That is, $F^{\prime}(0)=$ $0, F^{\prime \prime}(0)>0$. First, by solving formally the difference equation $x_{n+1}=G\left(x_{n}\right)=$ $x_{n}-x_{n}^{2}$, we estimate the asymptotic behavior $x_{n} \simeq n^{-1}, n \rightarrow \infty$. To estimate the asymptotic behavior of $n_{\delta}$, as $\delta \rightarrow 0$, we use, as above, the relation $2 \delta \simeq$ $\left(x_{n_{\delta}}-x_{n_{\delta+1}}\right)$. Since $x_{n}-x_{n+1}=F\left(x_{n}\right)=x_{n}^{2} \simeq n^{-2}$, we get that $n_{\delta} \simeq \delta^{-1 / 2}$, as $\delta \rightarrow 0$. Consequently, $x_{n_{\delta}} \simeq \delta^{1 / 2}$. we now have

$$
\left|T_{\delta}\right|=2 \delta n_{\delta} \simeq \delta^{1 / 2},\left|N_{\delta}\right|=x_{n_{\delta}}+2 \delta \simeq \delta^{1 / 2}, \delta \rightarrow 0 .
$$

Therefore, $\left|U_{\delta}\right| \simeq \delta^{1 / 2}, \delta \rightarrow 0$, and $\operatorname{dim}_{B} G=\frac{1}{2}$. All calculations are independent of the initial point $x_{0}$.

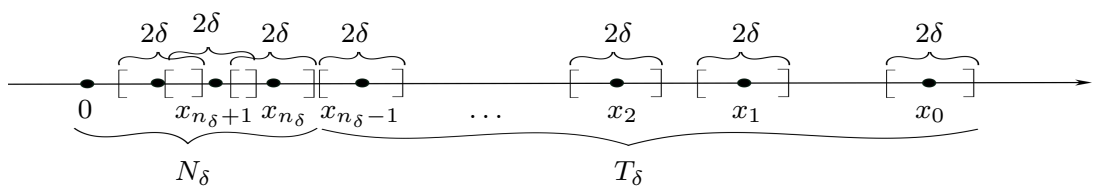

Figure 2: The $\delta$-neighborhood $U_{\delta}$ of $\mathcal{O}_{x_{0}}^{G}$ has two parts: the nucleus $N_{\delta}$, and the tail $T_{\delta}$. The tail $T_{\delta}$ contains all $(2 \delta)$-intervals of $U_{\delta}$ before they start to overlap at the point $x_{n_{\delta}}$.

Remark 1. In the hyperbolic case, the orbit $\mathcal{O}_{x_{0}}^{G}$ tends exponentially fast to the fixed point 0 . In Theorem 1, we make a distinction between the standard hyperbolic case and the degenerate hyperbolic case; the orbit $\mathcal{O}_{x_{0}}^{G}$ in the degenerate hyperbolic case tends to 0 faster than $\mathcal{O}_{x_{0}}^{G}$ in the standard hyperbolic case. Note that, by Theorem 1, the box dimension does not distinguish between standard and degenerate hyperbolic cases (in both cases, the box dimension is trivial). See e.g. [MRŽ12] for more details. 
Remark 2. Let $F(x, \lambda)$ be a smooth function in $(x, \lambda)$ near $\left(0, \lambda_{0}\right) \in \mathbb{R} \times \mathbb{R}^{p}$ such that the function $F\left(x, \lambda_{0}\right)$ satisfies the conditions of Theorem 1 . Let $G_{\lambda_{0}}(x)=x-F\left(x, \lambda_{0}\right)$. When $0 \leq \operatorname{dim}_{B} G_{\lambda_{0}}<1$, the Malgrange preparation theorem and Theorem 1 imply that $F(x, \lambda)$ has at most $\frac{1}{1-\operatorname{dim}_{B} G_{\lambda_{0}}}$ zeros (counting multiplicity) with respect to $x \sim 0$, for each $\lambda \sim \lambda_{0}$. Thus, in order to find out how many zeros the smooth $\lambda$-family $F(x, \lambda)$ has near $x=0$, it suffices to compute the box dimension of any orbit of the function $G_{\lambda_{0}}$.

Remark 3. In the study of the cyclicity of canard cycles one often encounters $\epsilon$-regularly smooth functions where $\epsilon \geq 0$ is the singular perturbation parameter (see e.g. [DMD05, DR07] or Section 4). A function $F(x, \mu, \epsilon)$, with $\mu \in \mathbb{R}^{p}$, is said to be $\epsilon$-regularly smooth in $(x, \mu)$ if $F$ is continuous in $(x, \mu, \epsilon)$, including $\epsilon=$ 0 , and all partial derivatives of $F$ with respect to $(x, \mu)$ exist and are continuous in $(x, \mu, \epsilon)$, including $\epsilon=0$. If $x=0$ is a zero of multiplicity $m$ of $F\left(x, \mu_{0}, 0\right)$, then $F(x, \mu, \epsilon)$ has at most $m$ zeros (counting multiplicity) with respect to $x \sim 0$, for each $\mu \sim \mu_{0}$ and $\epsilon \sim 0$. In fact, the $\epsilon$-regularly smoothness of the function $F(x, \mu, \epsilon)$ preserves $C^{\infty}$-stable properties of $F\left(x, \mu_{0}, 0\right)$ for a small $\epsilon$ (see e.g. [DMD05, DR07]). Again by Theorem 1, in order to find the number of zeros of $F(x, \mu, \epsilon)$ with respect to $x \sim 0$, for each $(\mu, \epsilon) \sim\left(\mu_{0}, 0\right)$, it is sufficient to compute the length of the $\delta$-neighborhood of an arbitrary but fixed orbit of the function $G(x)=x-F\left(x, \mu_{0}, 0\right)$ and compare it to $\delta, \delta^{\frac{1}{2}}, \delta^{\frac{1}{3}}, \ldots$ to obtain the box dimension of $G$.

\section{Definition of planar slow-fast models and state- ment of the results}

We consider slow-fast systems

$$
\left\{\begin{aligned}
\dot{x}= & y \\
\dot{y}= & -x y+\epsilon\left(\sum_{k=0}^{2 n-2} b_{k} x^{k}-x^{2 n-1}+x^{2 n} H(x, \mu)\right) \\
& +\epsilon y^{2} G(x, y, \mu),
\end{aligned}\right.
$$

where $\epsilon \sim 0$ is the singular perturbation parameter, $b=\left(b_{0}, b_{1}, \ldots, b_{2 n-2}\right) \sim$ $(0,0, \ldots, 0)$, with $n \geq 1, \mu \in M$, with $M$ a compact subset of $\mathbb{R}^{p}$, and where $H$ and $G$ are smooth functions. When $\epsilon=0$, the slow curve of (3) is given by $\{y=0\}$. The slow curve is normally attracting (resp. normally repelling) when $x>0$ (resp. $x<0$ ) and contains a nilpotent contact point at the origin $(x, y)=$ $(0,0)$. The fast orbits of the layer equation of (3) are parabolas $y=-\frac{x^{2}}{2}+C$ (see Fig. 3), and the dynamics of (3), with $\epsilon>0$ and $\epsilon \sim 0$, away from the slow curve, is similar to the dynamics of the layer equation. The slow dynamics of (3) along the slow curve (away from the contact point) is given by

$$
x^{\prime}=\frac{\sum_{k=0}^{2 n-2} b_{k} x^{k}-x^{2 n-1}+x^{2 n} H(x, \mu)}{x}, x \neq 0 .
$$

Let's explain how we get (4). Center manifolds of $(3)+0 \frac{\partial}{\partial \epsilon}$, at the normally hyperbolic singularity $(x, y, \epsilon)=(x, 0,0), x \neq 0$, are given by $y=\epsilon(d(x, b, \mu)+$ $O(\epsilon)$ ) where the function $d(x, b, \mu)$ is the right-hand side of (4). From this together with the first equation in (3) we obtain the dynamics inside the center 
manifolds, near the slow curve: $\dot{x}=\frac{d x}{d t}=\epsilon(d(x, b, \mu)+O(\epsilon))$. If we divide both sides of this equation by $\epsilon$ and let $\epsilon$ tend to zero, we obtain the slow dynamics (4): $x^{\prime}=\frac{d x}{d \tau}=\frac{d x}{\epsilon d t}=d(x, b, \mu)$. For more details about the definition of slow dynamics see e.g. [DR96, KS01].

In the limiting case, when $b=0$, the slow dynamics (4) can be written as $x^{\prime}=x^{2 n-2}(-1+x H(x, \mu))$, clearly pointing from the right to the left at least for $x \sim 0$ and $x \neq 0$ (i.e. $x^{\prime}<0$ for $x \sim 0$ and $x \neq 0$ ). Thus, it makes sense to study relaxation oscillations of (3) near canard cycles $\Gamma_{y}, y>0$, consisting of the fast orbit of the layer equation through the point $(0, y)$ on the $y$-axis, and the part of the slow curve between the $\alpha$-limit set and the $\omega$-limit set of the fast orbit (see Fig. 3). In studying the cyclicity of $\Gamma_{y}$, we essentially distinguish between the case where the slow dynamics is nonzero at the contact point $x=0$, so-called slow-fast Hopf case $(n=1, b=0)$, and the case where the slow dynamics has a zero of multiplicity $2,4,6, \ldots$, at the contact point $(n>1, b=0)$.

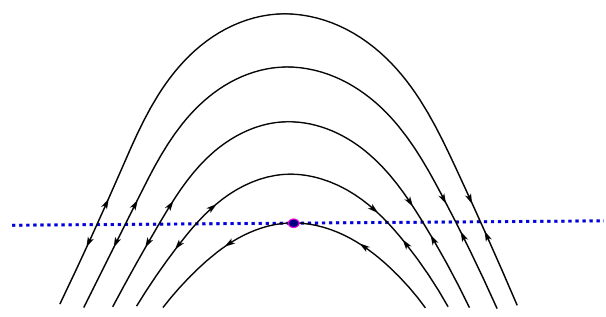

Figure 3: The layer equation of (3).

\subsection{Slow-fast Hopf case}

In this section we focus on the slow-fast system (3) with $n=1,\{\dot{x}=y, \dot{y}=$ $\left.-x y+\epsilon\left(b_{0}-x+x^{2} H(x, \mu)\right)+\epsilon y^{2} G(x, y, \mu)\right\}$, which we denote by $X_{\epsilon, b_{0}, \mu}^{H}$. Following [Dum11], $X_{\epsilon, b_{0}, \mu}^{H}$ has at $(x, y)=(0,0)$ a generic turning point.

We fix $y_{0}>0$ and $\mu_{0} \in M$ and study limit cycles of $X_{\epsilon, b_{0}, \mu}^{H}$ near the canard cycle $\Gamma_{y_{0}}$, for $\left(\epsilon, b_{0}, \mu\right) \sim\left(0,0, \mu_{0}\right)$. When $\mu=\mu_{0}$, we suppose the slow dynamics of $X_{\epsilon, b_{0}, \mu}^{H}$, given by $x^{\prime}=d(x, \mu):=-1+x H(x, \mu)$, is regular on $\left[\alpha_{0}\left(y_{0}\right), \omega_{0}\left(y_{0}\right)\right]$ with possible isolated (nonzero) singularities of even multiplicity positioned in the interval $] \alpha_{0}\left(y_{0}\right), \omega_{0}\left(y_{0}\right)\left[\right.$, where the $C^{\infty}$ diffeomorphism $\alpha_{0}(y)<0$ (resp. $\omega_{0}(y)>0$ ), with $y \sim y_{0}$, is the $x$-component of the $\alpha$-limit set (resp. the $\omega$-limit set) of the fast orbit contained in $\Gamma_{y}$ (clearly, we have $\alpha_{0}(y)=-\sqrt{2 y}$ and $\omega_{0}(y)=\sqrt{2 y}$ in our case). Thus, the slow dynamics $d\left(x, \mu_{0}\right)$ is negative on $\left[\alpha_{0}\left(y_{0}\right), \omega_{0}\left(y_{0}\right)\right]$, except at the singularities, near which passage is possible for $\mu \sim \mu_{0}$ and $\epsilon>0$ due to their even multiplicity. In [DMD08], the slow divergence integral $I(y, \mu)$ along the slow curve $\left[\alpha_{0}(y), \omega_{0}(y)\right]$ has been used as a tool for studying the cyclicity of $\Gamma_{y_{0}}$, where

$$
I(y, \mu)=\int_{\alpha_{0}(y)}^{\omega_{0}(y)} \frac{s d s}{d(s, \mu)},(y, \mu) \sim\left(y_{0}, \mu_{0}\right) .
$$

(The divergence of $X_{0, b_{0}, \mu}^{H}$ on the slow curve $\{y=0\}$ is $-x$ and $d \tau=\frac{d s}{d(s, \mu)}$.) When the slow dynamics $d\left(x, \mu_{0}\right)$ is regular on $\left[\alpha_{0}\left(y_{0}\right), \omega_{0}\left(y_{0}\right)\right]$, then the cyclicity 
of $\Gamma_{y_{0}}$ near $\mu=\mu_{0}$ is bounded from above by $1+$ the multiplicity of zero of $I\left(y, \mu_{0}\right)$ at $y=y_{0}$. When $d\left(x, \mu_{0}\right)$ has singularities in $] \alpha_{0}\left(y_{0}\right), \omega_{0}\left(y_{0}\right)[,(5)$ makes no sense, but the derivative $\frac{\partial I}{\partial y}$ is a well-defined smooth function near $(y, \mu)=$ $\left(y_{0}, \mu_{0}\right)$, and $2+$ the multiplicity of $\frac{\partial I}{\partial y}\left(y, \mu_{0}\right)$ at $y=y_{0}$ is an upper bound on the cyclicity of $\Gamma_{y_{0}}$ near $\mu=\mu_{0}$ (see [DMD08]).

Suppose that $d\left(x, \mu_{0}\right)$ is negative for each $x \in\left[\alpha_{0}\left(y_{0}\right), \omega_{0}\left(y_{0}\right)\right]$ and that $\Gamma_{y_{0}}$ is a balanced canard cycle, with $\mu=\mu_{0}$, i.e. $I\left(y_{0}, \mu_{0}\right)=0$ (for more details about definition of balanced canard cycles see e.g. [Dum11]). Since $\alpha_{0}$ and $\omega_{0}$ are smooth diffeomorphisms, the Implicit Function Theorem implies existence of a unique smooth function $G(y, \mu)$ near $(y, \mu)=\left(y_{0}, \mu_{0}\right)(G$ is a $\mu$-family of smooth diffeomorphisms with respect to $y$ ) such that $y_{0}$ is a fixed point of $G\left(y, \mu_{0}\right)$, i.e. $G\left(y_{0}, \mu_{0}\right)=y_{0}, \frac{\partial G}{\partial y}\left(y_{0}, \mu_{0}\right)=\frac{d\left(\alpha_{0}\left(y_{0}\right), \mu_{0}\right)}{d\left(\omega_{0}\left(y_{0}\right), \mu_{0}\right)}>0$ and

$$
\int_{\alpha_{0}(G(y, \mu))}^{\omega_{0}(y)} \frac{s d s}{d(s, \mu)}=0,(y, \mu) \sim\left(y_{0}, \mu_{0}\right) .
$$

We call $G$ a slow relation function (see [Dum11]). Since our goal is to apply Theorem 1 to the function $G_{\mu_{0}}(y):=G\left(y, \mu_{0}\right)$, we suppose that the orbit $\mathcal{O}_{y_{0}^{+}}^{G_{\mu_{0}}}=\left(y_{n}^{+}\right)_{n \geq 0}$ is decreasing and tends to $y_{0}$, for each $y_{0}^{+}>y_{0}, y_{0}^{+} \sim y_{0}$, or that the orbit $\mathcal{O}_{y_{0}^{-}}^{G_{\mu_{0}}}=\left(y_{n}^{-}\right)_{n \geq 0}$ is increasing and tends to $y_{0}$, for each $y_{0}^{-}<y_{0}$, $y_{0}^{-} \sim y_{0}$. More precisely, when $y_{0}$ is an asymptotically stable hyperbolic fixed point of $G_{\mu_{0}}$ (i.e. $0<G_{\mu_{0}}^{\prime}\left(y_{0}\right)<1$ ), both orbits, $\mathcal{O}_{y_{0}^{+}}^{G_{\mu_{0}}}$ and $\mathcal{O}_{y_{0}^{-}}^{G_{\mu_{0}}}$, tend monotonically to $y_{0}$. (Note that the "degenerate" hyperbolic case $\left(G_{\mu_{0}}^{\prime}\left(y_{0}\right)=0\right.$ ) is not possible.) If $G_{\mu_{0}}^{\prime}\left(y_{0}\right)>1$, the hyperbolic fixed point $y_{0}$ is unstable and consequently $\mathcal{O}_{y_{0}^{+}}^{G_{\mu_{0}}}$ and $\mathcal{O}_{y_{0}^{-}}^{G_{\mu_{0}}}$ don't converge to $y_{0}$; in this case we consider $-X_{\epsilon, b_{0}, \mu}^{H}$ (see Remark 4). Suppose now that $y_{0}$ is a nonhyperbolic fixed point of $G_{\mu_{0}}$ (i.e. $G_{\mu_{0}}^{\prime}\left(y_{0}\right)=1$ ). First, we assume $G_{\mu_{0}}$ has even multiplicity $2 l$ at $y=y_{0}$, for $l \geq 1$. If $G_{\mu_{0}}^{(2 l)}\left(y_{0}\right)<0$ (resp. $G_{\mu_{0}}^{(2 l)}\left(y_{0}\right)>0$ ), then the graph of $G_{\mu_{0}}$ is concave down (resp. concave up) and $\mathcal{O}_{y_{0}^{+}}^{G_{\mu_{0}}}\left(\right.$ resp. $\left.\mathcal{O}_{y_{0}^{-}}^{G_{\mu_{0}}}\right)$ tends to $y_{0}$. Assume now that $G_{\mu_{0}}$ has odd multiplicity $2 l+1$ at $y=y_{0}$, for $l \geq 1$ (i.e. $y=y_{0}$ is a point of inflection for $\left.G_{\mu_{0}}\right)$. If $G_{\mu_{0}}^{(2 l+1)}\left(y_{0}\right)<0$ (resp. $\left.G_{\mu_{0}}^{(2 l+1)}\left(y_{0}\right)>0\right)$ both orbits, $\mathcal{O}_{y_{0}^{+}}^{G_{\mu_{0}}}$ and $\mathcal{O}_{y_{0}^{-}}^{G_{\mu_{0}}}$, tend to $y_{0}$ (resp. $\mathcal{O}_{y_{0}^{+}}^{G_{\mu_{0}}}$ and $\mathcal{O}_{y_{0}^{-}}^{G_{\mu_{0}}}$ don't converge to $y_{0}$ (see Remark 4)).

We denote by $\operatorname{dim}_{B} G_{\mu_{0}}$ the box dimension of $\mathcal{O}_{y_{0}^{+}}^{G_{\mu_{0}}}$ or $\mathcal{O}_{y_{0}^{-}}^{G_{\mu_{0}}}$, depending on which one tends to $y_{0}$. Clearly, if both orbits converge to $y_{0}$, we have $\operatorname{dim}_{B} G_{\mu_{0}}=\operatorname{dim}_{B} \mathcal{O}_{y_{0}^{+}}^{G_{\mu_{0}}}=\operatorname{dim}_{B} \mathcal{O}_{y_{0}^{-}}^{G_{\mu_{0}}}$ (see Theorem 1).

Remark 4. When $G_{\mu_{0}}^{\prime}\left(y_{0}\right) \geq 1$, we can consider the vector field $-X_{\epsilon, b_{0}, \mu}^{H}$ and a $\mu$-family of smooth diffeomorphisms $\widetilde{G}_{\mu}(y)$, near $(y, \mu)=\left(y_{0}, \mu_{0}\right)$, such that $\widetilde{G}_{\mu_{0}}\left(y_{0}\right)=y_{0}$ and $\int_{\alpha_{0}(y)}^{\omega_{0}\left(\widetilde{G}_{\mu}(y)\right)} \frac{s d s}{d(s, \mu)}=0,(y, \mu) \sim\left(y_{0}, \mu_{0}\right)$, where $\alpha_{0}, \omega_{0}, d$ are defined above. It is straightforward to check that $\widetilde{G}_{\mu_{0}}^{\prime}\left(y_{0}\right)=\frac{1}{G_{\mu_{0}}^{\prime}\left(y_{0}\right)} \leq 1$ $\left(G_{\mu_{0}}^{-1}=\widetilde{G}_{\mu_{0}}\right)$. Thus, a hyperbolically unstable fixed point $y_{0}$ of $G_{\mu_{0}}$ becomes a hyperbolically stable fixed point of $\widetilde{G}_{\mu_{0}}$ and therefore $\mathcal{O}_{y_{0}^{ \pm}}^{\widetilde{G}_{\mu_{0}}}$ tend to $y_{0}$. On 
the other hand, if the multiplicity of $G_{\mu_{0}}$ is $2 l+1$ at $y=y_{0}$, for $l \geq 1$, and $G_{\mu_{0}}^{(2 l+1)}\left(y_{0}\right)>0$, then the multiplicity of $\widetilde{G}_{\mu_{0}}$ is $2 l+1$ at $y=y_{0}$ and $\widetilde{G}_{\mu_{0}}^{(2 l+1)}\left(y_{0}\right)<0$. Thus, $\mathcal{O}_{y_{0}^{ \pm}}^{\widetilde{G}_{\mu_{0}}}$ tend to $y_{0}$.

Since the origin is a slow-fast Hopf point, i.e. a generic turning point, closed orbits of $X_{\epsilon, b_{0}, \mu}^{H}$, close in the Hausdorff sense to $\Gamma_{y_{0}}$, can occur only if $\left(\epsilon, b_{0}\right)=$ $\left(\bar{\epsilon}^{2}, \bar{\epsilon} B_{0}\right)$, where $\bar{\epsilon}>0, \bar{\epsilon} \sim 0$ is a new singular perturbation parameter and where $B_{0} \sim 0$ is the so called (regular) breaking parameter. This can be proved using the family blow-up at the origin $(x, y, \epsilon)=(0,0,0)$ (for more details see e.g. [DR96, KS01, Dum11]). We define $X_{\bar{\epsilon}, B_{0}, \mu}:=X_{\bar{\epsilon}^{2}, \bar{\epsilon} B_{0}, \mu}^{H}$.

When there exist $\bar{\epsilon}_{0}>0$ and $B_{0}^{0}>0$ small, a neighborhood $V_{1}$ of $\mu_{0}$ in $\mu$-space and a (Hausdorff) neighborhood $V_{2}$ of $\Gamma_{y_{0}}$ so that the slow fast system $X_{\bar{\epsilon}, B_{0}, \mu}$ has at most $N$ limit cycles in $V_{2}$, for each $\left(\bar{\epsilon}, B_{0}, \mu\right) \in\left[0, \bar{\epsilon}_{0}\right] \times\left[-B_{0}^{0}, B_{0}^{0}\right] \times$ $V_{1}$, we say that the cyclicity of $\Gamma_{y_{0}}$ of $X_{\bar{\epsilon}, B_{0}, \mu}$, near $\left(\bar{\epsilon}, B_{0}, \mu\right)=\left(0,0, \mu_{0}\right)$, is bounded from above by $N$. The smallest $N$ with this property is the cyclicity of $\Gamma_{y_{0}}$ of $X_{\bar{\epsilon}, B_{0}, \mu}$, near $\left(\bar{\epsilon}, B_{0}, \mu\right)=\left(0,0, \mu_{0}\right)$.

Theorem 2. Let $y_{0}>0, \mu_{0} \in M$ and suppose the slow dynamics $d\left(x, \mu_{0}\right)$ is negative for $x \in\left[\alpha_{0}\left(y_{0}\right), \omega_{0}\left(y_{0}\right)\right]$. If the canard cycle $\Gamma_{y_{0}}$ is balanced at $\mu=\mu_{0}$, i.e. $I\left(y_{0}, \mu_{0}\right)=0$, and $0 \leq \operatorname{dim}_{B} G_{\mu_{0}}<1$ where the smooth diffeomorphism $G_{\mu_{0}}$ is defined in (6), then the cyclicity of $\Gamma_{y_{0}}$ of $X_{\bar{\epsilon}, B_{0}, \mu}$, near $\left(\bar{\epsilon}, B_{0}, \mu\right)=\left(0,0, \mu_{0}\right)$, is bounded from above by $\frac{2-\operatorname{dim}_{B} G_{\mu_{0}}}{1-\operatorname{dim}_{B} G_{\mu_{0}}}$.

Remark 5. Theorem 2 provides a useful tool for finding an upper bound on the number of limit cycles of $X_{\bar{\epsilon}, B_{0}, \mu}$ near $\Gamma_{y_{0}}$. Instead of working directly with the slow divergence integral $I(y, \mu)$, one can find one orbit of the slow relation function $G_{\mu_{0}}$ by using (6), and compute its box dimension.

Remark 6. Theorem 2 is also true in more general case when we deal with so called non-generic turning points in smooth planar slow-fast systems which satisfy Assumptions T0-T6 of [DMD05] (see also [DMD06]). A typical example of non-generic turning points is $\left\{\dot{x}=y-x^{2 n}, \dot{y}=\epsilon\left(b_{0}-x^{2 n-1}+O\left(x^{2 n}\right)\right)\right\}$, with $n \geq 1$ (when $n=1$, we deal with a slow-fast Hopf point at $(x, y)=(0,0)$ ). The slow dynamics along the slow curve $\left\{y=x^{2 n}\right\}$ is given by $x^{\prime}=-\frac{1}{2 n}+O(x)$. Like in the slow-fast Hopf case, we can introduce a regular breaking parameter $B_{0} \sim 0:\left(\epsilon, b_{0}\right)=\left(\bar{\epsilon}^{2 n}, \bar{\epsilon}^{2 n-1} B_{0}\right)$. If the slow dynamics is regular along the slow curve (this assumption is included in T0-T6), the slow divergence integral along the slow curve is well defined and we can introduce the slow relation function near balanced canard cycles by using an equation similar to (6). Note also that in the proof of Theorem 2 (Section 4.1) we use the results obtained in [DMD05].

Theorem 2 will be proved in Section 4.1.

Suppose now that the slow dynamics $d\left(x, \mu_{0}\right)$ is negative for each $x \in$ $\left[\alpha_{0}\left(\widetilde{y}_{0}\right), \omega_{0}\left(\widetilde{y}_{0}\right)\right]$, for some fixed $\widetilde{y}_{0}>y_{0}$. Following e.g. [Dum11], since $B_{0}$ is the breaking parameter, there exists a smooth function $B_{0}(\bar{\epsilon}, \mu)$, defined near $(\bar{\epsilon}, \mu)=\left(0, \mu_{0}\right), B_{0}(0, \mu)=0$, such that the system $X_{\bar{\epsilon}, B_{0}(\bar{\epsilon}, \mu), \mu}$ has a closed orbit, close to $\Gamma_{\widetilde{y}_{0}}$ and passing through the point $(x, y)=\left(0, \widetilde{y}_{0}\right)$, for each $(\bar{\epsilon}, \mu) \sim\left(0, \mu_{0}\right)$ and $\bar{\epsilon}>0$. We call $B_{0}=B_{0}(\bar{\epsilon}, \mu)$ the control curve.

First, we assume that $y_{0}$ is a hyperbolically stable fixed point of $G_{\mu_{0}}$ or, equivalently, $\operatorname{dim}_{B} G_{\mu_{0}}=0$. 
Theorem 3. (Hyperbolic fixed point) Let $\widetilde{y}_{0}>y_{0}>0, \mu_{0} \in M$ and assume the slow dynamics $d\left(x, \mu_{0}\right)$ is negative for $x \in\left[\alpha_{0}\left(\widetilde{y}_{0}\right), \omega_{0}\left(\widetilde{y}_{0}\right)\right]$. Assume furthermore that the canard cycle $\Gamma_{y_{0}}$ is balanced at $\mu=\mu_{0}$, i.e. $I\left(y_{0}, \mu_{0}\right)=0$, and that $\operatorname{dim}_{B} G_{\mu_{0}}=0$. Then the following statements are true:

1 The cyclicity of $\Gamma_{y_{0}}$ of $X_{\bar{\epsilon}, B_{0}, \mu}$, near $\left(\bar{\epsilon}, B_{0}, \mu\right)=\left(0,0, \mu_{0}\right)$, is equal to 2 , and, fixing $(\bar{\epsilon}, \mu)$, with $(\bar{\epsilon}, \mu) \sim\left(0, \mu_{0}\right)$ and $\bar{\epsilon}>0$, the $B_{0}$-family $X_{\bar{\epsilon}, B_{0}, \mu}$ contains a saddle-node bifurcation of limit cycles (Hausdorff) close to $\Gamma_{y_{0}}$.

2 If $B_{0}(\bar{\epsilon}, \mu)$ is the control curve, defined above, then the system $X_{\bar{\epsilon}, B_{0}(\bar{\epsilon}, \mu), \mu}$ has a unique limit cycle (Hausdorff) close to $\Gamma_{y_{0}}$, for each $(\bar{\epsilon}, \mu) \sim\left(0, \mu_{0}\right)$, $\bar{\epsilon}>0$. The limit cycle is hyperbolic and attracting.

Theorem 3 will be proved in Section 4.2.

Write $\mu=\left(\mu^{1}, \ldots, \mu^{p}\right)$ and $\mu_{0}=\left(\mu_{0}^{1}, \ldots, \mu_{0}^{p}\right)$. When $G_{\mu_{0}}\left(y_{0}\right)=y_{0}, G_{\mu_{0}}^{\prime}\left(y_{0}\right)=$ $1, G_{\mu_{0}}^{\prime \prime}\left(y_{0}\right) \neq 0$ and the function $\mu \rightarrow G_{\mu}\left(y_{0}\right)$ is a submersion at $\mu=\mu_{0}$ (for example, $\left.\left.\frac{\partial G_{\mu}}{\partial \mu^{1}}\right|_{\mu=\mu_{0}}\left(y_{0}\right) \neq 0\right)$, then, fixing $\left(\mu^{2}, \ldots, \mu^{p}\right) \sim\left(\mu_{0}^{2}, \ldots, \mu_{0}^{p}\right)$, the $\mu^{1}$ family $G_{\mu}, \mu^{1} \sim \mu_{0}^{1}$, contains a saddle-node bifurcation of fixed points close to $y_{0}$ (see e.g. [HSD13]).

Theorem 4. (Saddle-node bifurcation of fixed points) Let $\widetilde{y}_{0}>y_{0}>0, \mu_{0} \in M$ and assume the slow dynamics $d\left(x, \mu_{0}\right)$ is negative for $x \in\left[\alpha_{0}\left(\widetilde{y}_{0}\right), \omega_{0}\left(\widetilde{y}_{0}\right)\right]$. Assume furthermore that the canard cycle $\Gamma_{y_{0}}$ is balanced at $\mu=\mu_{0}$, i.e. $I\left(y_{0}, \mu_{0}\right)=0$. If $\operatorname{dim}_{B} G_{\mu_{0}}=\frac{1}{2}$ and the function $\mu \rightarrow G_{\mu}\left(y_{0}\right)$ is a submersion at $\mu=\mu_{0}$, then the following statements are true:

1 The cyclicity of $\Gamma_{y_{0}}$ of $X_{\bar{\epsilon}, B_{0}, \mu}$, near $\left(\bar{\epsilon}, B_{0}, \mu\right)=\left(0,0, \mu_{0}\right)$, is equal to 3 .

2 If $B_{0}(\bar{\epsilon}, \mu)$ is the control curve, defined above, then the cyclicity of $\Gamma_{y_{0}}$ of the system $X_{\bar{\epsilon}, B_{0}(\bar{\epsilon}, \mu), \mu}$, near $(\bar{\epsilon}, \mu)=\left(0, \mu_{0}\right)$, is equal to 2 . When $\left.\frac{\partial G_{\mu}}{\partial \mu^{j}}\right|_{\mu=\mu_{0}}\left(y_{0}\right) \neq 0$, for some $j \in\{1, \ldots, p\}$, then, for each $\bar{\epsilon} \sim 0, \bar{\epsilon}>0$, $\left(\mu^{1}, \ldots, \mu^{j-1}, \mu^{j+1}, \ldots, \mu^{p}\right) \sim\left(\mu_{0}^{1}, \ldots, \mu_{0}^{j-1}, \mu_{0}^{j+1}, \ldots, \mu_{0}^{p}\right)$, the $\mu^{j}$-family $X_{\bar{\epsilon}, B_{0}(\bar{\epsilon}, \mu), \mu}$, with $\mu^{j} \sim \mu_{0}^{j}$, undergoes a saddle-node bifurcation of limit cycles near $\Gamma_{y_{0}}$.

Theorem 4 will be proved in Section 4.3 .

Remark 7. The proof of Theorems 3-4 is based on Theorem 1, Lemma 1 and [Dum11]. Similar results can be proved for any generic Hopf breaking mechanism defined in [Dum11], not just the one studied here. Furthermore, the same can be proved for a generic jump breaking mechanism consisting of an attracting part of slow curve, a (generic) jump point, a fast orbit between this jump point and a second (generic) jump point, and a repelling part of the slow curve starting at the second jump point (for a precise definition of a generic jump breaking mechanism see [Dum11]). We point out that Theorem 4.3 and Theorem 4.6 of [Dum11], which we use in the proof of Theorems 3-4, have been proved both in the Hopf mechanism and the jump mechanism.

We finish this section with the following theorem.

Theorem 5. Let $y_{0}>0$ and $\mu_{0} \in M$. The following statements are true: 
1 If the slow dynamics $d\left(x, \mu_{0}\right)$ is negative for $x \in\left[\alpha_{0}\left(y_{0}\right), \omega_{0}\left(y_{0}\right)\right]$, the canard cycle $\Gamma_{y_{0}}$ is balanced at $\mu=\mu_{0}$, i.e. $I\left(y_{0}, \mu_{0}\right)=0$, and $0 \leq \operatorname{dim}_{B} G<$ 1 where $G(y):=y-I\left(y, \mu_{0}\right)$, then the cyclicity of $\Gamma_{y_{0}}$ of $X_{\bar{\epsilon}, B_{0}, \mu}$, near $\left(\bar{\epsilon}, B_{0}, \mu\right)=\left(0,0, \mu_{0}\right)$, is bounded by $\frac{2-\operatorname{dim}_{B} G}{1-\operatorname{dim}_{B} G}$.

2 If the slow dynamics $d\left(x, \mu_{0}\right)$ is negative for $x \in\left[\alpha_{0}\left(y_{0}\right), \omega_{0}\left(y_{0}\right)\right]$, except at the singularities, positioned in $] \alpha_{0}\left(y_{0}\right), \omega_{0}\left(y_{0}\right)\left[, \frac{\partial I}{\partial y}\left(y_{0}, \mu_{0}\right)=0\right.$ and $0 \leq$ $\operatorname{dim}_{B} G<1$ where $G(y):=y-\frac{\partial I}{\partial y}\left(y, \mu_{0}\right)$, then the cyclicity of $\Gamma_{y_{0}}$ of $X_{\bar{\epsilon}, B_{0}, \mu}$, near $\left(\bar{\epsilon}, B_{0}, \mu\right)=\left(0,0, \mu_{0}\right)$, is bounded by $\frac{3-2 \operatorname{dim}_{B} G}{1-\operatorname{dim}_{B} G}$.

Theorem 5 will be proved in Section 4.4.

Remark 8. Theorem 5(1) (resp. Theorem 5(2)) is true in the more general framework of [DMD05] (resp. [DMD08]). Note that in the proof of Theorem 5(2) (Section 4.4) we use results of [DMD08] that can be applied not only to the slow-fast Hopf case but also to a broader class of planar slow-fast systems (defined in [DMD08]) whose slow dynamics is regular with possible isolated singularities away from the contact point (see Theorem 2.5 of [DMD08]). For more details about the definition of that class of slow-fast systems we refer to [DMD08].

\subsection{Slow dynamics with a zero of order $2 n-2$ at the contact point $(n \geq 2)$}

In this section, we study limit cycles of (3), with $n \geq 2$, close to $\Gamma_{y_{0}}$, for $(\epsilon, b, \mu) \sim$ $\left(0,0, \mu_{0}\right)$. We suppose the slow dynamics of $(3)$, given by $x^{\prime}=d(x, \mu):=$ $x^{2 n-2}(-1+x H(x, \mu))$, is negative for $\mu=\mu_{0}, x \neq 0$ and $x \in\left[\alpha_{0}\left(y_{0}\right), \omega_{0}\left(y_{0}\right)\right]$, where $\alpha_{0}$ and $\omega_{0}$ are defined in Section 3.1. Like in Section 3.1, we define the slow divergence integral $I(y, \mu)$ along the slow curve $\left[\alpha_{0}(y), \omega_{0}(y)\right]$ by

$$
I(y, \mu)=\int_{\alpha_{0}(y)}^{\omega_{0}(y)} \frac{s d s}{d(s, \mu)},(y, \mu) \sim\left(y_{0}, \mu_{0}\right) .
$$

Even though the integral (7) makes no sense $\left(I\left(y, \mu_{0}\right)=\infty-\infty\right)$, we can study zeros of the well defined and smooth derivative $\frac{\partial I}{\partial y}(y, \mu)$, near $(y, \mu)=\left(y_{0}, \mu_{0}\right)$. It has been proven in [DMD10] $(n=2)$ and [DMD11a] $(n \geq 3)$ that $2+$ the multiplicity of $\frac{\partial I}{\partial y}\left(y, \mu_{0}\right)$ at $y=y_{0}$ is an upper bound on the number of limit cycles close to $\Gamma_{y_{0}}$. From this, together with Theorem 1, we obtain

Theorem 6. Let $n \geq 2, y_{0}>0$ and $\mu_{0} \in M$. If the slow dynamics $d\left(x, \mu_{0}\right)$ is negative for $x \neq 0$ and $x \in\left[\alpha_{0}\left(y_{0}\right), \omega_{0}\left(y_{0}\right)\right], \frac{\partial I}{\partial y}\left(y_{0}, \mu_{0}\right)=0$ and $0 \leq \operatorname{dim}_{B} G<1$ where $G(y):=y-\frac{\partial I}{\partial y}\left(y, \mu_{0}\right)$, then the cyclicity of $\Gamma_{y_{0}}$ of $(3)$, near $(\bar{\epsilon}, b, \mu)=$ $\left(0,0, \mu_{0}\right)$, is bounded by $\frac{3-2 \operatorname{dim}_{B} G}{1-\operatorname{dim}_{B} G}$.

Theorem 6 will be proved in Section 4.5.

Remark 9. The system (3), with $n \geq 2$, is a special case of the planar slow-fast systems whose slow dynamics has a zero of order $2 n-2$ at the turning point, studied in [DMD10, DMD11a], and the results of [DMD10, DMD11a] that we use in the proof of Theorem 6 have been proved for that large class of slow-fast systems. Thus, Theorem 6 can be proved in the more general framework of [DMD10, DMD11a]. 


\section{Proofs of Theorem 2-Theorem 6}

Let $y_{0}>0$ and $\mu_{0} \in M$. We denote by $X_{\epsilon, b, \mu}$ the vector field (3), with $n \geq 1$. The cyclicity of $\Gamma_{y_{0}}$ is usually studied using the difference map

$$
\Delta(y, \epsilon, b, \mu)=\Delta_{+}(y, \epsilon, b, \mu)-\Delta_{-}(y, \epsilon, b, \mu)
$$

defined near $(y, \epsilon, b, \mu)=\left(y_{0}, 0,0, \mu_{0}\right) . \Delta_{+}$(resp. $\left.\Delta_{-}\right)$is the transition map of $X_{\epsilon, b, \mu}$ (resp. $-X_{\epsilon, b, \mu}$ ) from a section $S_{1}=\{x=0\}$, parametrized by $y \sim y_{0}$, to a section $S_{2}=\{x=0\}$, parametrized by $y \sim 0$. Closed orbits of $X_{\epsilon, b, \mu}$, Hausdorff close to $\Gamma_{y_{0}}$, are represented by zeros of the difference map $\Delta$ with respect to $y \sim y_{0}$. It is more convenient to study the number of zeros of $\frac{\partial \Delta}{\partial y}$, or equivalently, the number of zeros of the divergence integral

$$
D(y, \epsilon, b, \mu)=\int_{\mathcal{O}^{+}(y, \epsilon, b, \mu)} \operatorname{div} X_{\epsilon, b, \mu} d t-\int_{\mathcal{O}^{-}(y, \epsilon, b, \mu)} \operatorname{div}\left(-X_{\epsilon, b, \mu}\right) d t
$$

and then use Rolle's theorem (see [DR96, DMD05, DMD08, DMD10, DMD11a]). $\mathcal{O}^{+}(y, \epsilon, b, \mu)$ (resp. $\left.\mathcal{O}^{-}(y, \epsilon, b, \mu)\right)$ is the orbit of $X_{\epsilon, b, \mu}\left(\right.$ resp. $\left.-X_{\epsilon, b, \mu}\right)$ from $(0, y) \in S_{1}$ to $S_{2}$.

We say that a smooth function $\psi_{1}(y, \mu)$ is $C^{\infty}$-contact equivalent to a smooth function $\psi_{2}(y, \mu)$ if there exists a strictly positive smooth function $\Psi(y, \mu)$ such that $\psi_{1}(y, \mu)=\Psi(y, \mu) \psi_{2}(y, \mu)$ (see e.g. [DR01, Dum11]). Clearly, if $\psi_{1}(y, \mu)$ and $\psi_{2}(y, \mu)$ are $C^{\infty}$-contact equivalent, then $y=y_{0}$ is a zero of multiplicity $m$ of $\psi_{1}\left(y, \mu_{0}\right)$ if and only if $y=y_{0}$ is a zero of multiplicity $m$ of $\psi_{2}\left(y, \mu_{0}\right)$. The following simple observation will be used in Sections 4.1-4.3.

Lemma 1. If $d\left(x, \mu_{0}\right)$ is negative for $x \in\left[\alpha_{0}\left(y_{0}\right), \omega_{0}\left(y_{0}\right)\right]$, then $I(y, \mu)$ is $C^{\infty}$ contact equivalent to $y-G(y, \mu)$, near $(y, \mu)=\left(y_{0}, \mu_{0}\right)$, where $d(x, \mu), I(y, \mu)$ and $G(y, \mu)$ are defined in Section 3.1.

Proof. From (5) and (6), it follows that

$I(y, \mu)=\int_{\alpha_{0}(y)}^{\omega_{0}(y)} \frac{s d s}{d(s, \mu)}=\left(\int_{\alpha_{0}(y)}^{\omega_{0}(y)}+\int_{\omega_{0}(y)}^{\alpha_{0}(G(y, \mu))}\right) \frac{s d s}{d(s, \mu)}=\int_{\alpha_{0}(y)}^{\alpha_{0}(G(y, \mu))} \frac{s d s}{d(s, \mu)}$.

Since the integrand $\frac{s}{d(s, \mu)}$ is positive for all $s \in\left[\alpha_{0}(y), \alpha_{0}(G(y, \mu))\right]$, and $\frac{\partial \alpha_{0}}{\partial y}<0$, The Fundamental Theorem of Calculus implies the statement.

\subsection{Proof of Theorem 2}

We suppose that $n=1$, i.e. the system $X_{\epsilon, b, \mu}$ has a slow-fast Hopf point at $(x, y)=(0,0)$, and we focus on $X_{\bar{\epsilon}, B_{0}, \mu}$, defined in Section 3.1. Since the slow dynamics of $X_{\bar{\epsilon}, B_{0}, \mu}$ is regular on $\left[\alpha_{0}\left(y_{0}\right), \omega_{0}\left(y_{0}\right)\right]$, for $\mu \sim \mu_{0}$, [DMD05] implies that the divergence integral (8) can be written as

$$
D\left(y, \bar{\epsilon}^{2}, \bar{\epsilon} B_{0}, \mu\right)=\frac{1}{\bar{\epsilon}^{2}}\left(I(y, \mu)+\Phi\left(y, \bar{\epsilon}, B_{0}, \mu\right)\right),\left(y, \bar{\epsilon}, B_{0}, \mu\right) \sim\left(y_{0}, 0,0, \mu_{0}\right)
$$

where $I(y, \mu)$ is the slow divergence integral along $\left[\alpha_{0}(y), \omega_{0}(y)\right]$ defined in (5), and $\Phi\left(y, \bar{\epsilon}, B_{0}, \mu\right)$ is $\bar{\epsilon}$-regularly smooth in $\left(y, B_{0}, \mu\right), \Phi\left(y, 0, B_{0}, \mu\right)=0$. More 
precisely, we have $\Phi\left(y, \bar{\epsilon}, B_{0}, \mu\right)=\phi_{1}\left(y, \bar{\epsilon}, B_{0}, \mu\right)+\phi_{2}\left(\bar{\epsilon}, B_{0}, \mu\right) \bar{\epsilon}^{2} \ln \bar{\epsilon}$, where $\phi_{1}$ and $\phi_{2}$ are smooth (including $\bar{\epsilon}=0$ ) and $\phi_{1}=O(\bar{\epsilon})$.

By Theorem 1, $y=y_{0}$ is a zero of multiplicity $m:=\frac{1}{1-\operatorname{dim}_{B} G_{\mu_{0}}}$ of $F\left(y, \mu_{0}\right)$, with $F(y, \mu)=y-G(y, \mu)$, where $G(y, \mu)$ is defined in (6). By Lemma 1, the slow divergence integral $I\left(y, \mu_{0}\right)$ has a zero of multiplicity $m$ at $y=y_{0}$, and using Remark 3 and (9), we obtain that $\frac{\partial \Delta}{\partial y}$ has at most $m$ zeros with respect to $y \sim y_{0}$, for each $\left(\bar{\epsilon}, B_{0}, \mu\right) \sim\left(0,0, \mu_{0}\right), \bar{\epsilon}>0$. Rolle's theorem implies now that the cyclicity of $\Gamma_{y_{0}}$ of $X_{\bar{\epsilon}, B_{0}, \mu}$, near $\left(\bar{\epsilon}, B_{0}, \mu\right)=\left(0,0, \mu_{0}\right)$, is bounded by $m+1=\frac{2-\operatorname{dim}_{B} G_{\mu_{0}}}{1-\operatorname{dim}_{B} G_{\mu_{0}}}$.

\subsection{Proof of Theorem 3}

We assume that the fixed point $y=y_{0}$ of $G_{\mu_{0}}$ is hyperbolically stable, i.e. $\operatorname{dim}_{B} G_{\mu_{0}}=0$. Thus, the function $F(y, \mu)=y-G(y, \mu)$ has a simple zero at $y=y_{0}$, for $\mu=\mu_{0}$. By Lemma 1 , the same is true for $I(y, \mu)$, and Theorem 3 follows directly from Theorem 4.3 of [Dum11]. We give a sketch of the proof of Theorem 3.

First we prove statement 2. We use the Poincaré-Bendixson theorem and the expression (6), which we call here the entry-exit relation. Let $(\bar{\epsilon}, \mu) \sim\left(0, \mu_{0}\right)$, with $\bar{\epsilon}>0$. Since $\operatorname{dim}_{B} G_{\mu_{0}}=0$, The Implicit Function Theorem implies existence of a unique (hyperbolically stable) fixed point $y(\mu) \sim y_{0}$ of $G(y, \mu)$, i.e. $G(y(\mu), \mu)=y(\mu)$. We choose $y_{0}^{-} \sim y_{0}$ and $y_{0}^{+} \sim y_{0}$ such that $y_{0}^{-}<y(\mu)<y_{0}^{+}$. Since $\Gamma_{y_{0}}$ is balanced at $\mu=\mu_{0}$, we deal with so-called tunneling behaviour along the slow curve $\left[\alpha_{0}\left(y_{0}\right), \omega_{0}\left(y_{0}\right)\right]$, and therefore we can use the entry-exit relation (6) to explain the behaviour of the orbits of $X_{\bar{\epsilon}, B_{0}(\bar{\epsilon}, \mu), \mu}$ near $\Gamma_{y_{0}}$ (see [Ben81b, Dum11]). More precisely, if we take an orbit of $X_{\bar{\epsilon}, B_{0}(\bar{\epsilon}, \mu), \mu}$, with starting point $(0, y) \in S_{1}, y \sim y_{0}$, it follows the fast orbit of $X_{0,0, \mu}$, then the slow curve $\left[\alpha_{0}(G(y, \mu)), \omega_{0}(y)\right]$, and a fast orbit again, and intersects the section $S_{1}$, in the limit $\bar{\epsilon} \rightarrow 0$, at the point $(0, G(y, \mu))$ (see Fig. 4). From this, together with the (asymptotic) stability of the fixed point $y(\mu)$, it follows that the orbits of $X_{\bar{\epsilon}, B_{0}(\bar{\epsilon}, \mu), \mu}$ with the initial points $\left(0, y_{0}^{-}\right)$and $\left(0, y_{0}^{+}\right)$are like in Fig. 4 . The Poincaré-Bendixson theorem implies now that $X_{\bar{\epsilon}, B_{0}(\bar{\epsilon}, \mu), \mu}$ has a hyperbolically attracting limit cycle Hausdorff close to $\Gamma_{y_{0}}$. For more details about the proof we refer to [Dum11].

We now give a sketch of the proof of statement 1 . Theorem 2 implies that the cyclicity of $\Gamma_{y_{0}}$ is bounded by 2 . It suffices to prove the existence of a saddle-node bifurcation of limit cycles near $\Gamma_{y_{0}}$.

It is well known ([Dum11]) that the closed orbits of the generic Hopf breaking mechanism $X_{\bar{\epsilon}, B_{0}, \mu}$ near $\Gamma_{y_{0}}$, with $\left(\bar{\epsilon}, B_{0}, \mu\right) \sim\left(0,0, \mu_{0}\right), \bar{\epsilon}>0$, are given by the solutions of

$$
\bar{B}_{0}=\exp \frac{1}{\bar{\epsilon}^{2}} L_{+}(y, \bar{\epsilon}, \mu)-\exp \frac{1}{\bar{\epsilon}^{2}} L_{-}(y, \bar{\epsilon}, \mu)
$$

where $\bar{B}_{0}=B_{0}-B_{0}(\bar{\epsilon}, \mu)$ is an adapted breaking parameter, $L_{ \pm}$are $\bar{\epsilon}$-regularly smooth in $(y, \mu), L_{+}(y, 0, \mu)=\int_{\omega_{0}(y)}^{0} \frac{-s d s}{d(s, \mu)}<0$ and $L_{-}(y, 0, \mu)=\int_{\alpha_{0}(y)}^{0} \frac{-s d s}{d(s, \mu)}<$ 0 . Note that

$$
L_{+}(y, 0, \mu)-L_{-}(y, 0, \mu)=I(y, \mu) .
$$




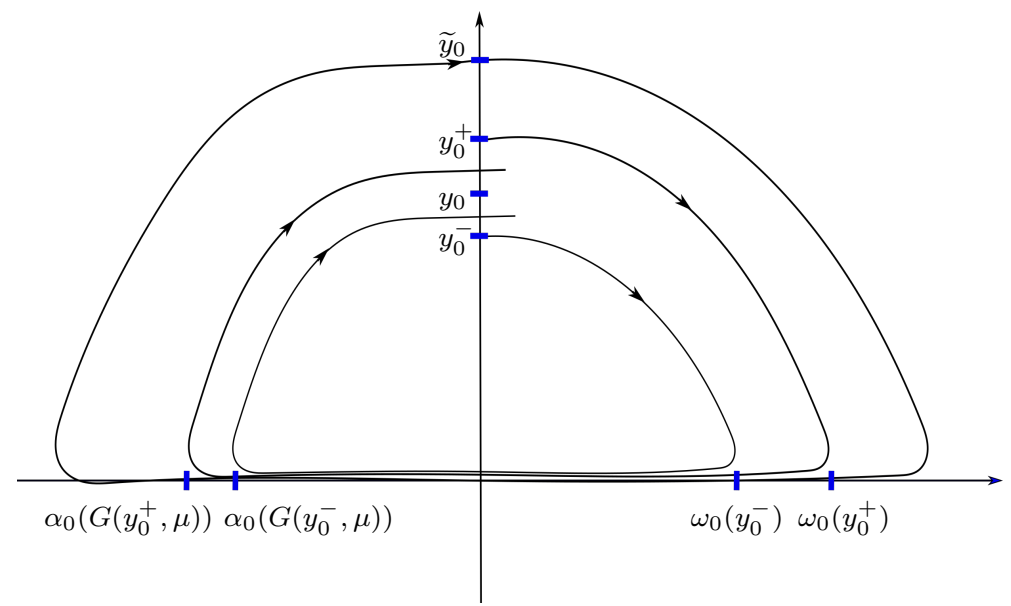

Figure 4: Orbits of $X_{\bar{\epsilon}, B_{0}(\bar{\epsilon}, \mu), \mu}$ governed by the entry-exit relation (6), for $(\bar{\epsilon}, \mu) \sim\left(0, \mu_{0}\right), \bar{\epsilon}>0$.

We denote by $\bar{B}_{0}(y, \bar{\epsilon}, \mu)$ the right side of (10). It can be easily seen that

$$
\frac{\partial \bar{B}_{0}}{\partial y}(y, \bar{\epsilon}, \mu)=\frac{1}{\bar{\epsilon}^{2}}\left(\frac{\partial L_{+}}{\partial y} \exp \frac{1}{\bar{\epsilon}^{2}} L_{+}-\frac{\partial L_{-}}{\partial y} \exp \frac{1}{\bar{\epsilon}^{2}} L_{-}\right),
$$

and

$$
\begin{aligned}
\frac{\partial^{2} \bar{B}_{0}}{\partial y^{2}}(y, \bar{\epsilon}, \mu)=\frac{1}{\bar{\epsilon}^{4}}( & \left(\frac{\partial L_{+}}{\partial y}\right)^{2} \exp \frac{1}{\bar{\epsilon}^{2}} L_{+}-\left(\frac{\partial L_{-}}{\partial y}\right)^{2} \exp \frac{1}{\bar{\epsilon}^{2}} L_{-} \\
& \left.+\bar{\epsilon}^{2} \frac{\partial^{2} L_{+}}{\partial y^{2}} \exp \frac{1}{\bar{\epsilon}^{2}} L_{+}-\bar{\epsilon}^{2} \frac{\partial^{2} L_{-}}{\partial y^{2}} \exp \frac{1}{\bar{\epsilon}^{2}} L_{-}\right) .
\end{aligned}
$$

If the right side of (11) is equal to zero, i.e.

$$
\frac{\partial L_{+}}{\partial y} \exp \frac{1}{\bar{\epsilon}^{2}} L_{+}=\frac{\partial L_{-}}{\partial y} \exp \frac{1}{\bar{\epsilon}^{2}} L_{-},
$$

the function $\frac{\partial^{2} \bar{B}_{0}}{\partial y^{2}}(y, \bar{\epsilon}, \mu)$ becomes

$$
\frac{\partial^{2} \bar{B}_{0}}{\partial y^{2}}(y, \bar{\epsilon}, \mu)=\frac{1}{\bar{\epsilon}^{4}} \exp \frac{1}{\bar{\epsilon}^{2}} L_{+}\left(\frac{\partial L_{+}}{\partial y}\left(\frac{\partial L_{+}}{\partial y}-\frac{\partial L_{-}}{\partial y}\right)+O\left(\bar{\epsilon}^{2}\right)\right),
$$

where $O\left(\bar{\epsilon}^{2}\right)$ is $\bar{\epsilon}$-regularly smooth in $(y, \mu)$. Note that $\frac{\partial L_{ \pm}}{\partial y}<0$ near $(\bar{\epsilon}, \mu)=$ $\left(0, \mu_{0}\right)$.

The equation (12) is equivalent to

$$
L_{+}-L_{-}=\bar{\epsilon}^{2} \ln \frac{\frac{\partial L_{-}}{\partial y}}{\frac{\partial L_{+}}{\partial y}} .
$$

Since $I(y, \mu)$ has a simple zero at $y=y_{0}$, for $\mu=\mu_{0}$, the Implicit Function Theorem implies that there exists a unique $\bar{\epsilon}$-regularly smooth solution $y=$ 
$y(\bar{\epsilon}, \mu) \sim y_{0}$ of (14). Clearly, (13) is nonzero for each $y=y(\bar{\epsilon}, \mu),(\bar{\epsilon}, \mu) \sim\left(0, \mu_{0}\right)$, $\bar{\epsilon}>0$, and therefore, $y=y(\bar{\epsilon}, \mu)$ represents a fold point for each $(\bar{\epsilon}, \mu) \sim$ $\left(0, \mu_{0}\right), \bar{\epsilon}>0$. Thus, the graph of $\bar{B}_{0}=\bar{B}_{0}(y, \bar{\epsilon}, \mu)$ is concave up/down, and consequently, for each $(\bar{\epsilon}, \mu) \sim\left(0, \mu_{0}\right), \bar{\epsilon}>0$, a saddle-node bifurcation of limit cycles takes place near $\Gamma_{y_{0}}$ as we vary $\bar{B}_{0}$.

Remark 10. Note that statement 2 can also be proved using (10), with $\bar{B}_{0}=$ 0, and The Implicit Function Theorem applied at the simple zero of the slow divergence integral. For more details see [Dum11].

\subsection{Proof of Theorem 4}

Since $\operatorname{dim}_{B} G_{\mu_{0}}=\frac{1}{2},(2)$ implies that $G_{\mu_{0}}$ has multiplicity 2 at $y=y_{0}$. Thus, $G_{\mu}$ contains a saddle-node bifurcation of fixed points near $(y, \mu)=\left(y_{0}, \mu_{0}\right)$, and we have $F_{\mu_{0}}\left(y_{0}\right)=0, F_{\mu_{0}}^{\prime}\left(y_{0}\right)=0, F_{\mu_{0}}^{\prime \prime}\left(y_{0}\right) \neq 0$, and $\mu \rightarrow F_{\mu}\left(y_{0}\right)$ is a submersion at $\mu=\mu_{0}$, where $F_{\mu}(y)=y-G_{\mu}(y)$. By Lemma 1 , the same is true for $I(y, \mu)$ and therefore $I(y, \mu)$ undergoes a saddle-node bifurcation of zeros at $(y, \mu)=\left(y_{0}, \mu_{0}\right)$. Statements 1 and 2 follow now directly from Theorem 4.6 of [Dum11]. For the sake of completeness, we sketch the proof of the statements.

First we prove statement 2 . When $B_{0}=B_{0}(\bar{\epsilon}, \mu)\left(\bar{B}_{0}=0\right)$, the equation (10) is equivalent to

$$
0=L_{+}(y, \bar{\epsilon}, \mu)-L_{-}(y, \bar{\epsilon}, \mu)=I(y, \mu)+O(\bar{\epsilon}),
$$

where $O(\bar{\epsilon})$ is $\bar{\epsilon}$-regularly smooth in $(y, \mu)$. Since $I(y, \mu)$ has a zero of multiplicity 2 at $y=y_{0}$, for $\mu=\mu_{0}$, Remark 3 and (15) imply that $\Gamma_{y_{0}}$ can produce at most 2 limit cycles in the family $X_{\bar{\epsilon}, B_{0}(\bar{\epsilon}, \mu), \mu}$. The rest of statement 2 follows from the fact that the saddle-node bifurcation of zeros is stable under the perturbations given in (15).

Let's prove statement 1 . Theorem 2 and $\operatorname{dim}_{B} G_{\mu_{0}}=\frac{1}{2}$ imply that $\Gamma_{y_{0}}$ can produce at most 3 limit cycles in $X_{\bar{\epsilon}, B_{0}, \mu}$. Since the function $\mu \rightarrow I\left(y_{0}, \mu\right)$ is a submersion at $\mu=\mu_{0}$, without loss of generality, we can suppose that $\frac{\partial I}{\partial \mu^{1}}\left(y_{0}, \mu_{0}\right) \neq 0$. (In the rest of the proof we don't need the parameter $\left(\mu^{2}, \ldots, \mu^{p}\right)$ and we can write $\left(\mu^{2}, \ldots, \mu^{p}\right)=\left(\mu_{0}^{2}, \ldots, \mu_{0}^{p}\right)$.) This and the fact that $I\left(y, \mu_{0}^{1}\right)$ has a zero of multiplicity 2 at $y=y_{0}$ imply that $I\left(y, \mu^{1}\right)$ is $C^{\infty}$-contact equivalent at $\left(y_{0}, \mu_{0}^{1}\right)$ to the elementary catastrophy (of codimension 2$) \pm Y^{2}+\lambda\left(\mu^{1}\right)$, where $Y\left(y, \mu^{1}\right)$ is a $\mu^{1}$-family of smooth local diffeomorphisms at $y=y_{0}$, with $\mu^{1} \sim \mu_{0}^{1}, Y\left(y_{0}, \mu_{0}^{1}\right)=0$ and where $\lambda\left(\mu^{1}\right)$ is a smooth local diffeomorphism near $\mu^{1}=\mu_{0}^{1}$ (see The Malgrange preparation theorem or Proposition 4.2 of [Dum11]). Theorem 4.6(2) of [Dum11] implies now that $X_{\bar{\epsilon}, B_{0}, \mu}$ has a limit cycle of multiplicity 3 , Hausdorff close to $\Gamma_{y_{0}}$, unfolded in an elementary catastrophy of codimension 3 .

\subsection{Proof of Theorem 5}

Theorem 5(1) follows from Theorem 2 and Lemma 1. Let's prove Theorem $5(2)$. Suppose that the slow dynamics $x^{\prime}=d\left(x, \mu_{0}\right)$ satisfies conditions of Theorem 5(2). Then Theorem 2.5 of [DMD08] tells us that the cyclicity of $\Gamma_{y_{0}}$ of $X_{\bar{\epsilon}, B_{0}, \mu}$ near $\left(\bar{\epsilon}, B_{0}, \mu\right)=(0,0,0)$ is bounded by $2+m$, where $m$ is the multiplicity of $\frac{\partial I}{\partial y}\left(y, \mu_{0}\right)$ at $y=y_{0}$. In fact, it has been proved in [DMD08] that $\bar{\epsilon}^{2} \frac{\partial D}{\partial y}\left(y, \bar{\epsilon}^{2}, \bar{\epsilon} B_{0}, \mu\right)=\frac{\partial I}{\partial y}(y, \mu)+O(\bar{\epsilon})$, with $O(\bar{\epsilon}) \bar{\epsilon}$-regularly smooth in $\left(y, B_{0}, \mu\right)$. 
Theorem 2.5 of [DMD08] follows now from Rolle's theorem and the smoothness of $O(\bar{\epsilon})$.

If $\frac{\partial I}{\partial y}\left(y_{0}, \mu_{0}\right)=0$, Theorem 1 implies that $m=\frac{1}{1-\operatorname{dim}_{B} G}$, with $G(y)=$ $y-\frac{\partial I}{\partial y}\left(y, \mu_{0}\right)$.

\subsection{Proof of Theorem 6}

As explained in Section 3.2, Theorem 6 follows from Theorem 1 and [DMD10, DMD11a] (see also Section 4.4).

\section{References}

[Ben81a] É. Benoît. Chasse au canard. II. Tunnels-entonnoirs-peignes. Collect. Math., 32(2):77-97, 1981.

[Ben81b] É. Benoit. Équations différentielles: relation entrée-sortie. C. $R$. Acad. Sci. Paris Sér. I Math., 293(5):293-296, 1981.

[DMD05] P. De Maesschalck and F. Dumortier. Time analysis and entryexit relation near planar turning points. J. Differential Equations, 215(2):225-267, 2005.

[DMD06] P. De Maesschalck and F. Dumortier. Canard solutions at nongeneric turning points. Trans. Amer. Math. Soc., 358(5):2291-2334 (electronic), 2006.

[DMD08] P. De Maesschalck and F. Dumortier. Canard cycles in the presence of slow dynamics with singularities. Proc. Roy. Soc. Edinburgh Sect. A, 138(2):265-299, 2008.

[DMD10] P. De Maesschalck and F. Dumortier. Singular perturbations and vanishing passage through a turning point. J. Differential Equations, 248(9):2294-2328, 2010.

[DMD11a] P. De Maesschalck and F. Dumortier. Detectable canard cycles with singular slow dynamics of any order at the turning point. Discrete Contin. Dyn. Syst., 29(1):109-140, 2011.

[DMD11b] P. De Maesschalck and F. Dumortier. Slow-fast Bogdanov-Takens bifurcations. J. Differential Equations, 250(2):1000-1025, 2011.

[DMDR11] P. De Maesschalck, F. Dumortier, and R. Roussarie. Cyclicity of common slow-fast cycles. Indag. Math. (N.S.), 22(3-4):165-206, 2011.

[DR96] F. Dumortier and R. Roussarie. Canard cycles and center manifolds. Mem. Amer. Math. Soc., 121(577):x+100, 1996. With an appendix by Li Chengzhi.

[DR01] F. Dumortier and R. Roussarie. Multiple canard cycles in generalized Liénard equations. J. Differential Equations, 174(1):1-29, 2001. 
[DR07] F. Dumortier and R. Roussarie. Canard cycles with two breaking parameters. Discrete Contin. Dyn. Syst., 17(4):787-806, 2007.

[DR09] F. Dumortier and R. Roussarie. Birth of canard cycles. Discrete Contin. Dyn. Syst. Ser. S, 2(4):723-781, 2009.

[Dum11] F. Dumortier. Slow divergence integral and balanced canard solutions. Qual. Theory Dyn. Syst., 10(1):65-85, 2011.

[EŽŽ07] N. Elezović, V. Županović, and D. Žubrinić. Box dimension of trajectories of some discrete dynamical systems. Chaos Solitons Fractals, 34(2):244-252, 2007.

[Fal90] K. Falconer. Fractal geometry. John Wiley and Sons, Ltd., Chichester, 1990. Mathematical foundations and applications.

[Fed69] H. Federer. Geometric measure theory. Die Grundlehren der mathematischen Wissenschaften, Band 153. Springer-Verlag New York Inc., New York, 1969.

[HDMD13] R. Huzak, P. De Maesschalck, and F. Dumortier. Limit cycles in slow-fast codimension 3 saddle and elliptic bifurcations. J. Differential Equations, 255(11):4012-4051, 2013.

[HDMD14] R. Huzak, P. De Maesschalck, and F. Dumortier. Primary birth of canard cycles in slow-fast codimension 3 elliptic bifurcations. Communications on Pure and Applied Analysis, 13(6):2641-2673, 2014.

[HSD13] M. W. Hirsch, S. Smale, and R. L. Devaney. Differential equations, dynamical systems, and an introduction to chaos. Elsevier/Academic Press, Amsterdam, third edition, 2013.

[Huz16] R. Huzak. Cyclicity of the origin in slow-fast codimension 3 saddle and elliptic bifurcations. Discrete Contin. Dyn. Syst., 36(1):171$215,2016$.

[Huz17] R. Huzak. Regular and slow-fast codimension 4 saddle-node bifurcations. J. Differential Equations, 262(2):1119-1154, 2017.

[KP99] S. G. Krantz and H. R. Parks. The geometry of domains in space. Birkhäuser Advanced Texts: Basler Lehrbücher. [Birkhäuser Advanced Texts: Basel Textbooks]. Birkhäuser Boston, Inc., Boston, MA, 1999.

[KS01] M. Krupa and P. Szmolyan. Relaxation oscillation and canard explosion. J. Differential Equations, 174(2):312-368, 2001.

[Mat95] P. Mattila. Geometry of sets and measures in Euclidean spaces, volume 44 of Cambridge Studies in Advanced Mathematics. Cambridge University Press, Cambridge, 1995. Fractals and rectifiability.

[MKKR94] E. F. Mishchenko, Yu. S. Kolesov, A. Yu. Kolesov, and N. Kh. Rozov. Asymptotic methods in singularly perturbed systems. Monographs in Contemporary Mathematics. Consultants Bureau, New York, 1994. Translated from the Russian by Irene Aleksanova. 
[MRŽ12] P. Mardešić, M. Resman, and V. Županović. Multiplicity of fixed points and growth of $\varepsilon$-neighborhoods of orbits. J. Differential Equations, 253(8):2493-2514, 2012.

[Tri95] C. Tricot. Curves and fractal dimension. Springer-Verlag, New York, 1995. With a foreword by Michel Mendès France, Translated from the 1993 French original.

[ŽŽ08] D. Žubrinić and V. Županović. Poincaré map in fractal analysis of spiral trajectories of planar vector fields. Bull. Belg. Math. Soc. Simon Stevin, 15(5, Dynamics in perturbations):947-960, 2008. 